\title{
Molécules indétectables par chromatographie en phase gazeuse
}

\section{Undetectable molecules in gas chromatography}

LUC HUMBERT*, Michel LHERMTTTE

Service de Toxicologie et Génopathies, CHRU de Lille - 59037 LILLE

*:Auteur à qui adresser la correspondance : Luc HUMBERT, Service de Toxicologie et Génopathies, CHRU de Lille, Hôpital Calmette, Bd du Prof. J. Leclercq - 59037 LILLE - FRANCE Tél : 0320444950 - Fax : 0320444729 - E-mail : 1-humbert@chru-lille.fr

(Reçu le 16 février 2005 ; accepté après modifications le 2 avril 2005)

\section{RÉSUMÉ}

La chromatographie en phase gazeuse (CPG) et la chromatographie liquide de haute performance (CLHP) sont les deux méthodes séparatives les plus employées dans les laboratoires de toxicologie. La CLHP possède un avantage important, elle permet de séparer n'importe quel type de molécule à̀ condition qu'elle soit soluble. La CPG est destinée aux substances volatiles, leurs détections sont possibles grâce à un grand nombre de détecteurs très sensibles (ionisation de flamme, thermoionique, capture d'électrons, ...), elle est couplable très aisément à un spectromètre de masse sans avoir recours à une interface. L'une des limitations majeures de la CPG tient à la faible volatilité d'un grand nombre de molécules due soit à un haut poids moléculaire (en général > $500 \mathrm{Da}$ ) ou à leur capacité à créer des liaisons dipôle-dipôle ou hydrogène qui élèvent de façon importante leur point d'ébullition. Pour rendre détectable certaines d'entre elles il est possible de modifier leur nature physicochimique en réalisant l'une des nombreuses méthodes de dérivations existantes qui permettent par greffage d'un groupement chimique d'abaisser leur point d'ébullition. Il existe en outre une thermolabilité de certaines molécules en présence de la température élevée de l'injecteur. Il est parfois possible d'atténuer les problèmes de thermodénaturation de certains composés en utilisant une injection de type "on column » qui introduit directement l'échantillon en tête de colonne analytique sans le vaporiser Cet article détaille certains des aspects théoriques et explique certains des mécanismes qui interviennent dans l'analyse par CPG.

\section{MOTS-CLES}

Chromatographie en phase gazeuse, volatilité, molécule apolaire, thermodénaturation.

\section{SUMMARY}

Gas chromatography (GC) and high perfornance liquid chromatography (HPLC) are the two separative methods most employed in the laboratories of toxicology. The HPLC has a significant advantage, it makes it possible to separate any type of molecule provided that it is soluble. The GC is intended for the volatile substances, their detections are possible with to a great number of very sensitive detectors (flame ionization, thermionic, electron capture...), it is connecting very easily with a mass spectrometer without having use to an interface. One of the major limitations of the CPG is due to the low volatility of a great number of molecules due by a high molecular weight (in general $>500$ Da) or with their capacity to create links dipole-dipole or hydrogen which raise in a significant way their point of boiling. To make detectable some of then it is possible to modify their physico-chemical nature by carrying out one of the many methods of existing derivations which make it possible by grafting of a chemical group to lower their point of boiling. There is moreover a thermolability of some molecules in the presence of the high temperature of the injector: It is also possible to attenuate the problems of thermodenaturation of some compounds by using an injection of the type "on column" which directly introduces the sample at the head of column analytical without vaporizing it. This article details some of the theoretical aspects and explains some of the mechanisms which intervene in the analysis by $C P G$.

\section{KEY-WORDS}

Gas chromatography, volatility, non-polar molecule, thermodenaturation. 


\section{Introduction}

Les principaux outils analytiques dont dispose actuellement le toxicologue se répartissent en deux grandes familles. La première correspond aux méthodes immunologiques qui sont largement employées. Elles ont pour principaux avantages d'être automatisables, rapides, ne nécessitant pour la plupart d'aucune préparation d'échantillon et d'être accessibles à l'ensemble des laboratoires. Elles ont pour principaux inconvénients leurs coûts et peuvent donner des résultats faussement positifs ou négatifs par manque de spécificité. La seconde correspond aux méthodes séparatives qui sont essentiellement des méthodes chromatographiques basées sur le partage des solutés entre deux phases, l'une mobile et l'autre stationnaire. On distingue la chromatographie liquide de haute performance (CLHP), si la phase mobile est un liquide et la chromatographie en phase gazeuse (CPG) si la phase mobile est un gaz. Ces méthodes existent depuis de très nombreuses années et les appareils n'ont cessé d'évoluer dans tous leurs composants : injecteur, détecteur (de façon considérable) mais également dans le choix et la nature de la phase stationnaire. Cependant pour être analysable par ces méthodes, les constantes physicochimiques de chaque molécule vont être déterminantes. En effet toutes les molécules ne peuvent être analysées sur l'ensemble de ces systèmes analytiques existants $(1,2)$. Une molécule ne peut par exemple être analysée en CLHP que si elle est soluble dans la phase mobile du système mais également capable si la détection est en UV d'absorber la lumière UV, or certains médicaments ne remplissent pas ces conditions (acide valproïque, méprobamate, ...). Concernant la CPG, les raisons entraînant une non détection sont plus nombreuses, en effet un premier pré-requis est indispensable, il faut que la molécule soit vaporisable. Or beaucoup de xénobiotiques ne le sont pas ou sont thermodégradables. Cette article recense les causes de cette non détection et aborde les moyens qui permettent dans certaines conditions de rendre ces molécules vaporisables. Elle rapporte également des molécules qui ne sont pas détectables par chromatographie en phase gazeuse.

\section{Principe de la méthode}

La CPG est une méthode de séparation sur colonne de substances volatiles (ou rendues volatiles) véhiculées par un gaz inerte appelé gaz vecteur. La nature de la colonne conditionne le type de chromatographie (partage ou adsorption). Le choix de la colonne sera fonction des propriétés physico-chimiques des composés à séparer. Un chromatographe en phase gazeuse est constitué d'un injecteur, d'un four (dont la température est programmable) contenant la colonne et d'un détecteur (figure 1).

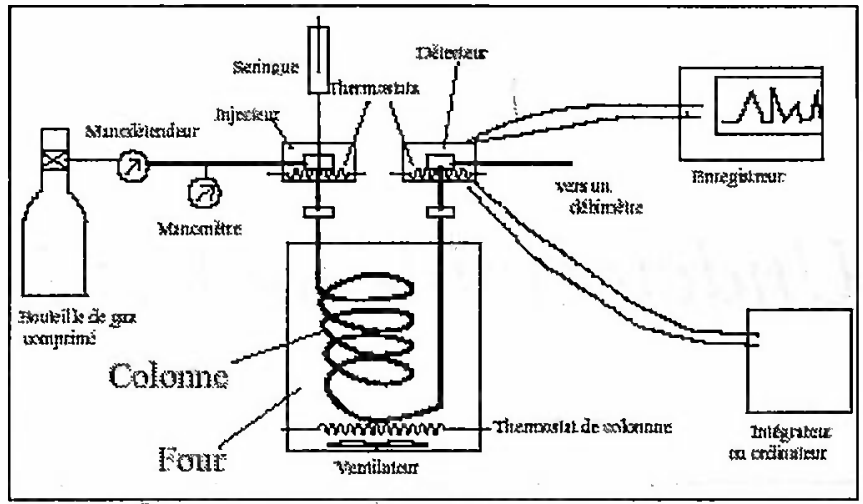

Figure I : Schéma général simplifié d'un chromatographe en phase gazeuse.

\section{Injecteur}

Relié à la colonne et obturé par un septum il permet d'introduire l'échantillon à analyser dissout dans un solvant organique. Sa température doit être supérieure d'environ $20^{\circ} \mathrm{C}$ à la température du produit le moins volatile. Le volume injecté est faible (quelques microlitres). L'injecteur le plus couramment utilisé est l'injecteur dit «split/splitless » (figure 2). Suivant l'échantillon à analyser, il est possible au moment de l'injection d'ouvrir ou pas une vanne qui permettra la propulsion vers la colonne de la totalité ou d'une partie de l'échantillon vaporisé. Parmi les différents injecteurs il faut citer un injecteur dit « on column » qui permet d'introduire l'échantillon directement sur la colonne analytique et de minorer substantiellement les phénomènes de thermodénaturation qui affectent certains composés.

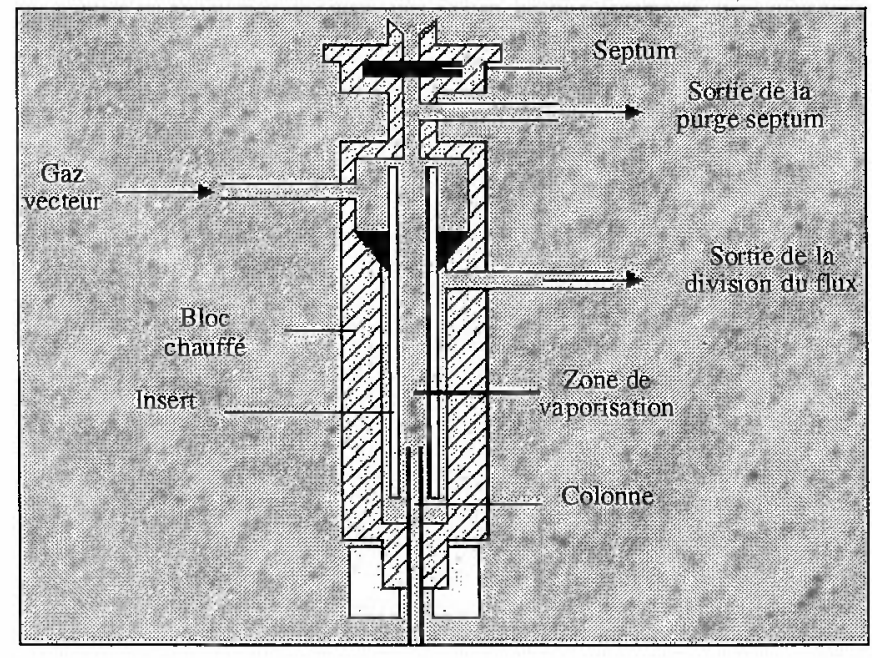

Figure 2 : Injecteur "split/splitless". 


\section{Four}

Le four est une enceinte thermostatée, sa programmation en température est possible jusqu'à environ $450^{\circ} \mathrm{C}$, il contient la colonne reliée d'une part à l'injecteur et de l'autre part au détecteur. C'est la nature de la phase stationnaire contenue dans la colonne analytique qui sera le facteur limitant dans cette programmation. En effet, suivant la nature de celle-ci la stabilité thermique du greffage est différent. Pour les phases stationnaires de type apolaire, il est préconisé des températures maximales comprises entre 320 et $350^{\circ} \mathrm{C}$ et entre 200 à $250^{\circ} \mathrm{C}$ pour les phases polaires.

\section{Détecteur}

Il permet de mettre en évidence le passage des différentes molécules séparées par la colonne. La détection peut être basée sur des techniques de mesures différentes. Le choix du détecteur va être un paramètre déterminant pour la limite de détection de l'analyse. Les concentrations à déterminer en toxicologie pourront être extrêmement faibles suivant la nature du xénobiotique et le contexte d'administration (suivi thérapeutique, tentative d'autolyse, soumission chimique, ...). Sa spécificité sera aussi un critère à prendre en compte dans son choix, certaines analyses, faites dans un contexte médicolégal, devront être réalisées avec les détecteurs de référence que sont les spectromètres de masse.

\section{Détecteur à ionisation de flamme}

C'est un détecteur très répandu, il ne donne de réponse qu'aux composés organiques. Sous l'effet d'un champ électrostatique, il se forme des ions carbone de charge positive qui sont précipités sur une électrode où ils créent un courant d'ionisation que l'on amplifie. Sur un enregistreur, on obtient par conséquent un signal proportionnel au débit-masse du soluté dans le détecteur

\section{Détecteur thermoionique}

Une pastille de sel alcalin est placée au contact de la flamme. La sensibilité est améliorée grandement (facteur 10 à 100) pour les molécules contenant un atome de phosphore ou d'azote.

\section{Détecteur à capture d'électrons}

Une source envoie des électrons libres dans le détecteur. Quand ce détecteur est traversé par des substances ayant une affinité pour les électrons libres, il se produit des ions qui, comme pour le détecteur à ionisation de flamme, dans le champ électrostatique existant, sont recueillis par une électrode et forment un courant d'ionisation que l'on amplifie. Sa sensibilité est très grande en particulier pour les substances renfermant des atomes d'halogène.

\section{Spectromètres de masse}

Les couplages CPG/SM ont pris au fil des années une part de plus en plus importante dans l'analyse par CPG. Le mode d'ionisation le plus répandue est le mode d'ionisation par impact électronique (IE), qui grâce aux bombardements par des électrons de haute énergie $(70 \mathrm{eV})$ permet d'obtenir des spectres de fragmentation caractérisant les molécules (spectres compilables dans des librairies). Ce mode de détection est plus sensible et peut parfois encore être amélioré en utilisant l'ionisation chimique (en mode positif ou négatif). Ce mode d'ionisation plus doux évite une fragmentation trop important et permet d'obtenir l'ion moléculaire (le plus souvent protoné ou déprotoné). Les propriétés physicochimiques de certaines molécules permettent un très bon rendement d'ionisation, améliorant considérablement la sensibilité de détection. C'est notamment le cas, en mode d'ionisation chimique négatif $(\mathrm{NCI})$ pour certaines benzodiazépines ( 3 ), les corticoïdes (4), ainsi que toutes les molécules qui sont dérivables par perfluoroalkylation (5). La détection en mode massemasse (triple quadripôle ou piège à ions) permet d'améliorer cette limite de détection des molécules en abaissant de façon importante le bruit de fond du système analytique $(6,7)$.

\section{Les couplages et leurs domaines d'appli- cations}

Les limites des différents couplages chromatographiques avec détection par spectrométrie de masse (SM) (figure 3) sont documentées dans la littérature. Le domaine d'application du couplage CPG/SM est beaucoup plus restreint que les couplages LC/SM dont le domaine ne cesse de s'étendre notamment avec l'émergence d'interfaces de plus en plus performantes (récemment a été introduit sur le marché une interface ionisant grâce à des photons permettant l'ionisation de molécules plus apolaires). La CPG/SM s'adresse à des molécules dont la nature est plutôt apolaire et d'un poids moléculaire inférieur à environ 500 Daltons. Un autre phénomène restreint encore ce domaine il s'agit de la thermodénaturation que subissent certaines molécules alors même que leurs propriétés physico-chimiques permettent des analyses par CPG/SM. La CPG s'applique donc à des échantillons gazeux ou susceptibles d'être vaporisés sans décomposition dans l'injecteur, sont a priori exclues les substances polaires, les substances thermolabiles et les substances de haut poids moléculaire. Ces différents points seront abordés et discutés. 


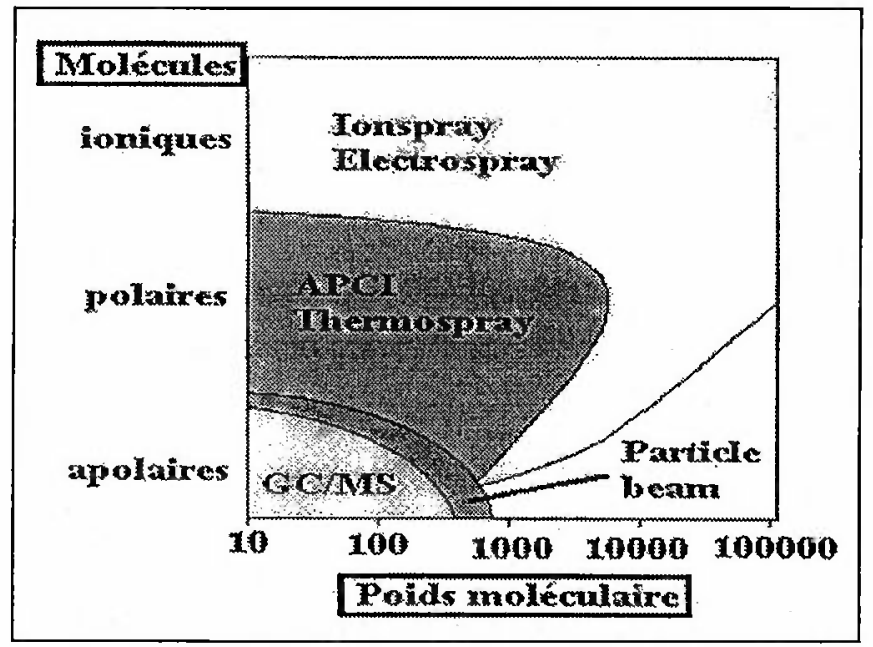

Figure 3 : Domaines d'applications des couplages chromatographiques avec la spectrométrie de masse.

\section{Notions théoriques}

A une température donnée, tout liquide pur est en équilibre avec sa phase vapeur (figure 4). Dans les solutions liquides, les molécules entrent en contact avec d'autres molécules. On observe alors un certain nombre d'interactions d'origine électrostatique produisant des forces intermoléculaires attractives entre les molécules neutres qui sont responsables de la formation des liquides et des solides à température ambiante.

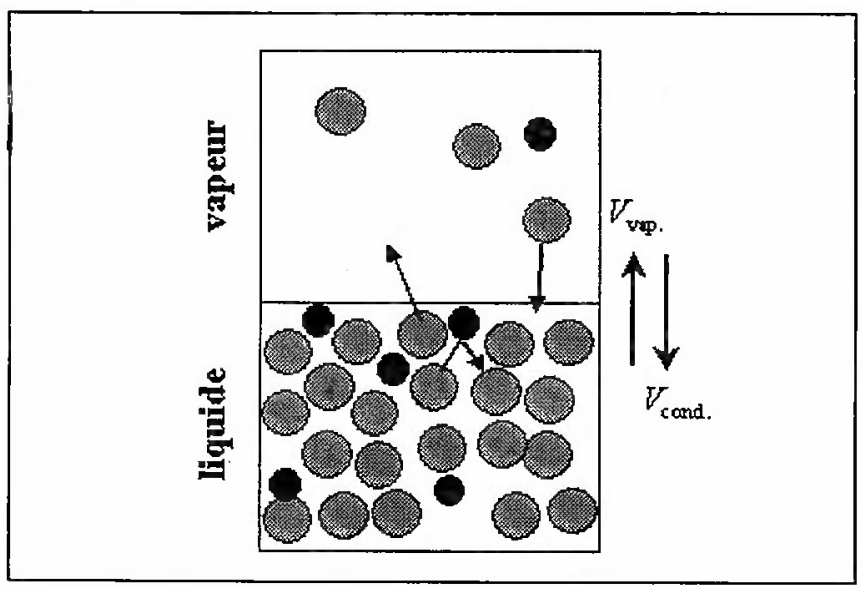

Figure 4 : Équilibre des molécules entre l'état vapeur et l'état liquide.

\section{L'échelle de polarité}

Cette échelle classe les molécules en trois catégories : polaire, apolaire et ionique.

Une substance polaire est une molécule à l'intérieur de laquelle les charges ne sont pas réparties de manière homogène. En principe, toute molécule polaire peut former des liaisons hydrogène avec les molécules d'eau. On dit qu'elle est hydrophile. Toute molécule hydrophile est soluble dans l'eau (hydrosoluble).

Une substance apolaire (dont aucune liaison ne montre de polarité suffisante, et qui ne possède pas de moment dipolaire ni de charge) est insoluble dans l'eau. On dit aussi qu'elle est hydrophobe.

Cela explique que l'eau soit liquide à la température ambiante (une molécule d'eau est capable de se lier avec trois autres molécules d'eau par des liaisons hydrogène), alors que le méthane $\left(\mathrm{CH}_{4}\right)$, molécule apolaire la plus simple est gazeux et n'a pas la capacité de former des liaisons hydrogène.

Une substance ionique crée une liaison ionique qui résulte de l'attraction entre une espèce positive (cation) et une espèce négative (anion). La stabilité de la liaison est assurée par l'interaction électrostatique, ces molécules sont les plus polaires et sont $\mathrm{pH}$ dépendant.

\section{Les interactions dipôle - dipôle}

L'attraction ou la répulsion entre molécules est due à différents effets :

- interactions entre molécules polaires (moments permanents) : effet Keesom.

- interactions entre moment permanent et un dipôle induit (moment qui n'existe pas naturellement pour une molécule mais qui est induit par un élément extérieur) : effet Debye.

- interactions entre moment dipolaire instantané (dû au déplacement des électrons) et moment induit : effet London.

C'est ainsi que l'on explique que deux molécules simples qui ont la même masse ( $56 \mathrm{~g})$ : la propanone et le butane ont des points d'ébullition différents.

Point d'ébullition de la propanone $\left(\mathrm{CH}_{3}\right)_{2} \mathrm{C}=\mathrm{O}:+56^{\circ} \mathrm{C}$ Point d'ébullition du butane $\mathrm{CH}_{3}-\mathrm{CH}_{2}-\mathrm{CH}_{2}-\mathrm{CH}_{3}:-0,5^{\circ} \mathrm{C}$ Cet écart important entre les points d'ébullition est imputable aux interactions dipôle-dipôle entre les molécules de propanone, interactions qu'il faut d'abord détruire pour pouvoir vaporiser la molécule (1 à 10 $\mathrm{kJ} / \mathrm{mol})$.

\section{Liaison hydrogène}

Il s'agit du cas particulier d'interaction dipôle-dipôle. Ce type de liaison prend naissance quand un atome d'hydrogène (atome de petite taille électropositif) est associé à un hétéroatome électronégatif (fluor, oxygène, azote, ...).

Les propriétés physico-chimiques d'une molécule sont fortement influencées par ce type de liaison. Par 
exemple, le point d'ébullition du méthanol est beaucoup plus élevé que celui de l'éthane :

Point d'ébullition de l'éthane $\mathrm{CH}_{3}-\mathrm{CH}_{3}(30 \mathrm{~g} / \mathrm{mol}):-89^{\circ} \mathrm{C}$ Point d'ébullition du méthanol $\mathrm{CH}_{3}-\mathrm{OH}(32 \mathrm{~g} / \mathrm{mol}):+64^{\circ} \mathrm{C}$ Toutes ces interactions peuvent intervenir également dans la formation de liaisons intramoléculaires qui abaissent les constantes physiques comparativement aux molécules, ayant la même formule brute, associées par des liaisons intermoléculaires (Figure 5). Elles interviennent aussi dans les dimérisations des acides carboxyliques, ces liaisons étant responsables d'une augmentation des constantes physiques.

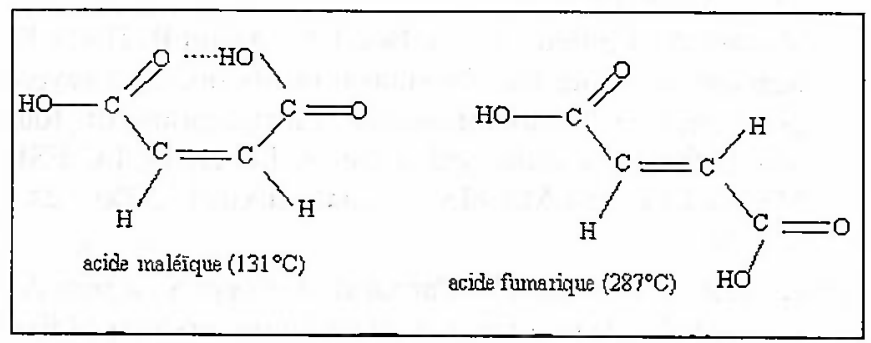

Figure 5 : Structures chimiques et points d'ébullitions des acides maléique et fumarique.

\section{Point d'ébullition}

Le point d'ébullition au sein d'une même famille dépend de la capacité des composés à créer des liaisons hydrogène entre eux ou avec le milieu dans lequel ils se trouvent. Le cas de la famille des opiacés est rapporté dans le tableau I. Le remplacement d'une des deux fonctions hydroxylées de la morphine par un groupement méthoxime (codéine) diminue de $100^{\circ} \mathrm{C}$ le point d'ébullition, phénomène amplifié encore s'il s'agit d'un groupement éthyl (codéthyline)

\section{Méthodes de dérivation}

Lorsque les composés ne sont pas volatils, on peut abaisser leur point d'ébullition en dérivant quantitativement et rapidement les groupements chimiques à l'ori-

Tableau I : Point d'ébullition des opiacés.

\begin{tabular}{|c|c|}
\hline Molécule & Point d'ébullition \\
\hline Morphine & 254 à $256^{\circ} \mathrm{C}$ avec décomposition \\
Codéine & 154 à $158^{\circ} \mathrm{C}$ \\
Codéthyline & $123{ }^{\circ} \mathrm{C}$ avec décomposition \\
Pholcodine & $99^{\circ} \mathrm{C}$ \\
Diacétylmorphine (hérö̈ne) & $170^{\circ} \mathrm{C}$ \\
\hline
\end{tabular}

gine de leur point d'ébullition élevé. Ces méthodes permettent d'augmenter la volatilité, la stabilité thermique et la symétrie chromatographique du composé parent. Elles sont nombreuses et sont largement utilisées en toxicologie : la méthylation qui consiste à remplacer un hydrogène labile par un groupement méthyle, la silylation substitue de la même manière un groupement triméthylsilyl (TMS) très couramment utilisé en $\mathrm{CPG} / \mathrm{SM}$, mais il en existe bien d'autres comme les perfluoroalkylations, la méthoximation.

\section{Thermodénaturation}

Seules des petites molécules de masse moléculaire inférieure à environ $200 \mathrm{~g} / \mathrm{mol}$ ont un point d'ébullition suffisamment bas pour que la vaporisation ne s'accompagne pas d'une décomposition thermique : alcanes, esters, cétones, alcools, amines (à l'exclusion de molécules chargées, de polyalcools, de sucres, des peptides). Pour les substances les plus stables thermiquement $\left(\mathrm{Teb}<250^{\circ} \mathrm{C}\right)$, la masse molaire peut atteindre 1000 $\mathrm{g} / \mathrm{mol}$, mais les cas sont rares.

De nombreux exemples de formation de produits de dégradations sont décrits (8) : décarboxylation de molécules possédant des fonctions carboxyliques, réarrangement avec perte d'une molécule d'eau (bis desalkylflurazépam), perte d'un groupement fonctionnel (formaldéhyde, ammoniac). Les pesticides issus d'urée substituée (diuron, métobromuron) se transforment sous l'effet de la température en isocyantes et/ou anilines (9). L'ibuprofène est un composé qui subit d'importantes dégradations sous l'effet de la température (10). Certains auteurs dosent la zopiclone par CPG/SM en suivant son produit de dégradation le 2 amino 5chloropyridine (11). Les anticancéreux comme le méthotrexate ne sont quantifiables que grâce aux kits immunologiques ou par couplage CLHP $(12,13)$. Certains de ces composés issus d'une thermodégradation sont parfois analysables en CPG et possèdent un spectre de masse de référence.

\section{Les molécules de haut poids moléculaire}

Un grand nombre de xénobiotiques ont un poids supérieur à la $500 \mathrm{Da}$, valeur couramment donnée comme limite supérieure des molécules détectables en CPG. C'est le cas des hormones (hormone de croissance (14), insuline (15)), des immunosuppresseurs (tacrolimus (16), sirolimus $(17,18)$, everolimus). Les venins d'animaux (scorpions, araignées, poissons, serpents) possèdent des poids moléculaires élevés et sont analysés grâce à des couplages CLHP/SM et CLPH/SM/SM (19-22). Certains végétaux supérieurs produisent ou sécrètent des substances toxiques pouvant induire des 
intoxications graves (23). Pour ne citer que quelques exemples : la digitale pourpre $(24,25)$ contient un grand nombre de digitaliques (digoxine, digitoxine, gitoxine), le charbon à glu (26) (atractyloside, ...), des champignons avec par exemple l'amanitine (27) ou la phalloïdine. Dans un travail compilant plus de 8000 poisons potentiels (28) 542 molécules ont un poids moléculaire supérieur à 500 Daltons, seulement 4 d'entre elles ont un spectre de masse (decamethrine, penfluridol, mirex, réserpine) et 6 possèdent un spectre de masse d'un produit de dégradation (piperacilline, bromadiolone, gliquidone, metofenazate, amiodarone, etiroxate). Les techniques de CLHP sont les seuls utilisables pour analyser les molécules de haut poids moléculaire.

\section{Conclusion}

La CPG est une méthode très largement employée dans les laboratoires de toxicologie. Son couplage à la spectrométrie de masse en fait depuis longtemps une méthode de référence. Ses limitations sont liées essentiellement aux propriétés physico-chimiques des molécules à analyser, leurs faibles volatilités ou leurs thermodénaturations nécessitent, quand leurs structures s'y prêtent, d'avoir recours à une ou des méthodes de dérivation qui rallongent le temps de préparation des échantillons. Les molécules de poids moléculaires élevés (supérieur en général à 500 Daltons) ne sont pas volatiles et ne sont pas analysables par ce type de chromatographie mais en CLHP qui prend une place de plus en plus importante notamment grâce à l'évolution permanente du couplage à la spectrométrie de masse (optimisation des interfaces, processus d'ionisation). Ces deux méthodes restent complémentaires et sont très utiles pour la recherche et/ou le dosage de xénobiotiques dont les structures chimiques sont extrêmement

\section{Références}

1. Drummer O. H. Chromatographic screening techniques in systematic toxicological analysis, J. Chromatogr. $1999 ; 733: 27-45$.

2. Levillain P. Place des méthodes séparatives dans le screening toxicologique d'urgence. Toxicorama. $1998 ; 10$ : 132-139.

3. Cirimele V., Kintz P., Mangin P. Determination of chronic flunitrazepam abuse by hair analysis using GC-MSNCI. J. Anal. Toxicol. 1996 ; 20 : 596-8.

4. Xiao X., McCalley D. Quantitative analysis of estrogens in human urine using gas chromatography /negative chemical ionisation mass spectrometry. Rapid Commun. Mass Spectrom. $2000 ; 14: 1991-2001$.
5. Maurer H.H., Kraemer T., Kratzsch C., Peters F.T., Weber AA. Negative ion chemical ionization gas chromatography-mass spectrometry and atmospheric pressure chemical ionization liquid chromatography-mass spectrometry of low-dosed and/or polar drugs in plasma. Ther. Drug. Monit. 2002 ; 24 : 117-24.

6. Kintz P., Cirimele V., Jamey C., Ludes B. Testing for GHB in hair by GC/MS/MS after a single exposure. Application to document sexual assault. J. Forensic. Sci. $2003 ; 48: 195-200$.

7. Gaillard Y., Pepin G. Testing hair for pharmaceuticals. J. Chromatogr. B. $1999 ; 733: 231-46$.

8. Pfleger K., Maurer H.H., Weber A. Mass spectral and GC data of drugs, poisons, pesticides, polluants and their metabolites. Second, revised and enlarged edition, VHC 1992 edition pp 21-24.

9. Turcảnt A., Cailleux A., Le Bouil A., Allain P., Harry P., Renault A. Acute metobromuron poisoning with severe associated methemoglobinemia. Identification of four metabolites in plasma and urine by LC-DAD, LC-ESIMS, and LC-ESI-MS-MS. J. Anal. Toxicol. $2000 ; 24$ : 157-64.

10. Gabriele C., Posocco V., Parodi B., Cafaggi S., Alzati A., Bignardi $G$. identification of degradation products of ibuprofen arising from oxidative and thermal treatments. J. of Pharmaceutical and biomedical analysis. $2002 ; 30$ : 499-509.

11. Galloway J.H., Marsh I.D., Newton C.M., Forrest A.R. A method for the rapid detection of the zopiclone degradation product 2-amino-5-chloropyridine. Sci. Justice. $1999 ; 39: 253-6$.

12. Dervieux T., Orentas Lein D., Marcelletti J., Pischel K., Smith K., Walsh M., Richerson R. HPLC determination of erythrocyte methotrexate polyglutamates after lowdose methotrexate therapy in patients with rheumatoid arthritis. Clin. Chem. $2003 ; 49: 1632-41$.

13. Widemann B.C., Sung E., Anderson L., Salzer W.L., Balis F.M., Monitjo K.S., McCully C., Hawkins M., Adamson P.C. Pharmacokinetics and metabolism of the methotrexate metabolite 2, 4-diamino-N(10)-methylpteroic acid. J. Pharmacol. Exp. Ther. $2000 ; 294$ : 894-901.

14. De Kock S.S., Rodgers J.P., Swanepoel B.C. Growth hormone abuse in the horse: preliminary assessment of a mass spectrometric procedure for IGF-1 identification and quantitation. Rapid Commun Mass Spectrom. 2001 ; $15: 1191-7$.

15. Darby S.M., Miller M.L., Allen R.O., LeBeau M. A mass spectrometric method for quantitation of intact insulin in blood samples. J. Anal. Toxicol. 2001 ; 25 : 8-14.

16. Taylor P.J., Jones A., Balderson G.A., Lynch S.V., Norris R.L., Pond S.M. Sensitive, specific quantitative analysis of tacrolimus (FK506) in blood by liquid chromatography-electrospray tandem mass spectrometry. Clin. Chem. $1996 ; 42: 279-85$.

17.Deters M., Kirchner G., Resch K., Kaever V. Simultaneous quantification of sirolimus, everolimus, tacrolimus and cyclosporine by liquid chromatographymass spectrometry (LC-MS). Clin. Chem. Lab. Med. $2002 ; 40: 28-92$. 
18. Volosov A., Napoli K.L., Soldin S.J. Simultaneous simple and fast quantification of three major immunosuppressants by liquid chromatography-tandem massspectrometry. Clin. Biochem. 2001 ; 34 : 285-90.

19. Legros C., Celerier M.L., Henry M., Guette C. Nanospray analysis of the venom of the tarantula Theraphosa leblondi: a powerful method for direct venom mass fingerprinting and toxin sequencing. Rapid Commun Mass Spectrom. 2004 ; 18 : 1024-32.

20. Nozawa A., Tsuji K., Ishida H. Implication of brevetoxin $\mathrm{B} 1$ and $\mathrm{PbTx}-3$ in neurotoxic shellfish poisoning in New Zealand by isolation and quantitative determination with liquid chromatography-tandem mass spectrometry. Toxicon. $2003 ; 42: 91-103$.

21.Itagaki Y., Fujita T., Naoki H., Yasuhara T., Andriantsiferana M., Nakajima T. Detection of new spider toxins from a Nephilengys borbonica venom gland using on-line mu-column HPLC continuous flow (FRIT) FAB LC/MS and MS/MS. Nat. Toxins. 1997 ; 5 : 1-13.

22. Fry B.G., Lumsden N.G., Wuster W., Wickramaratna J.C., Hodgson W.C., Kini R.M. Isolation of a neurotoxin (alpha-colubritoxin) from a nonvenomous colubrid: evidence for early origin of venom in snakes. J. Mol. Evol. $2003 ; 57: 446-52$.

23. Gaillard Y., Cheze M., Pepin G. Human main plant poisoning : revue of the litterature. Ann. Biol. Clin. (Paris). $2001 ; 59: 764-5$.
24. Plum J., Daldrup T. Detection of digoxin, digitoxin, their cardioactive metabolites and derivatives by high-performance liquid chromatography and high-performance liquid chromatography-radioimmunoassay. J. Chromatogr. $1986 ; 377: 221-31$.

25. Lacassie E., Gaulier J.M., Ragot S., Marquet P., Lachatre G. Dosages sériques et urinaires d'hétérosides cardiotoniques par LC-ES-MS, dans le cas d'intoxication non mortel par la digitalique pourpre. Toxicorama. 1999 ; $11: 110-116$.

26. Steenkamp P.A., Harding N.M., van Heerden F.R., van Wyk B.E. Determination of atractyloside in Callilepis laureola using solid-phase extraction and liquid chromatography-atmospheric pressure ionisation mass spectrometry. J. Chromatogr. A. 2004 ; 1058 : 15 -62.

27. Maurer H.H., Schmitt C.J., Weber A.A., Kraemer T. Validated electrospray liquid chromatographic-mass spectrometric assay for the determination of the mushroom toxins alpha- and beta-amanitin in urine after immunoaffinity extraction. J. Chromatogr. B. 2000 ; 748: 125-35.

28. Pfle.ger K., Maurer H.H., Weber A. Mass spectral and GC data of drugs, poisons, pesticides, polluants and their metabolites. Second, revised and enlarged edition, VHC 1992 edition pp 425-562. 